\title{
Brás Cubas, a Sepse e as Evidências: Reflexões sobre a Surviving Sepsis Campaign
}

\author{
Brás Cubas, Sepsis and the Evidence: \\ Reflections on the Surviving Sepsis Campaign
}

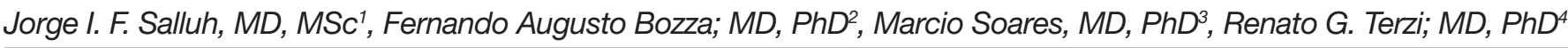

Brás Cubas em suas memórias póstumas nos conta como, apesar da invenção de um medicamento maravilhoso para os males do sofrimento humano - o Emplasto Brás Cubas - morreu da própria invenção; maravilhado com a sua idéia, descuidou da sua saúde. Preocupa-nos que hoje em meio à profusão de adventos tecnológicos e intervenções farmacológicas com evidências científicas heterogêneas, os intensivistas que cuidam de sepse possam vir a sofrer do mesmo mau de Brás Cubas, que maravilhado com o remédio esqueceu da saúde.

A sepse representa um problema clínico de grande relevância, devido as suas altas prevalência e letalidade, e ao custo elevado1. Sua incidência anual tem aumentado de maneira preocupante nas últimas décadas, sendo estimada nos Estados Unidos da América, em 1995, em 750.000 casos que causaram 215.000 mortes, a um custo anual de 16,7 bilhões de dólares². Apesar dos constantes avanços obtidos na terapêutica de suporte, assim como na antibioticoterapia, sua

1. Médico do Centro de Tratamento Intensivo do Instituto Nacional de Câncer, e do Hospital Barra D'Or. Rio de Janeiro, RJ; Mestre em Clínica Médica/Pneumologia pela UFRJ; Título de Especialista em Medicina Intensiva pela AMIB.

2. Médico do Instituto de Pesquisas Clínicas Evandro Chagas - Fundação Oswaldo Cruz, Rio de Janeiro. Doutor em Biologia Celular e Molecular. Título de Especialista em Medicina Intensiva pela AMIB.

3. Médico do Centro de Tratamento Intensivo do Instituto Nacional de Câncer, Rio de Janeiro, RJ; Mestre e Doutor em Clínica Médica pela UFRJ; Título de Especialista em Medicina Intensiva pela AMIB. 4. Professor Titular de Cirurgia da UNICAMP, Título de Especialista em Medicina Intensiva pela AMIB.

Endereço para correspondência:

Dr. Jorge I. F. Salluh

Instituto Nacional de Câncer - INCA

Centro de Tratamento Intensivo

Praça Cruz Vermelha, $23-10^{\circ}$ Andar

20230-130 Rio de Janeiro, RJ

Fone: (21) 2506-6120; Fax: (21) 2294-8620

E-mail: jorgesalluh@yahoo.com.br

(C)Associação de Medicina Intensiva Brasileira, 2006 mortalidade continua sendo extremamente elevada, variando nas formas mais graves, entre $40 \%$ e $80 \%$, sendo assim a principal causa de morte nas unidades de terapia intensiva ${ }^{2-5}$.

Estudos epidemiológicos recentes se ocuparam de diferentes aspectos da epidemiologia clínica da sepse. Angus e col. ${ }^{2}$ analisaram 6.621 .559 internações hospitalares no ano de 1995 em 847 hospitais e identificaram 192.980 casos de sepse grave. Neste estudo, a incidência de sepse foi estimada em 751.000 casos por ano nos EUA (300 casos por 100.000 habitantes ou 2,26 casos por 100 internações hospitalares), sendo que em torno de $383.000(51,1 \%)$ destes receberam cuidados intensivos. A letalidade hospitalar foi $28,6 \%$ e a letalidade na UTI foi $34,1 \%{ }^{2}$.

Martin e col. ${ }^{6}$ publicaram um estudo recente onde analisaram dados de 750 milhões de internações hospitalares nos EUA entre 1979 e 2000, identificando 10.319.418 casos de sepse, com importante incremento na incidência do diagnóstico de sepse ao, longo destes 22 anos. A incidência que era de 82,7 casos $/ 100.000$ habitantes em 1979, saltou para 240,4/100.000 em 2000. No entanto, a letalidade apresentou um declínio significativo, de $27,8 \%$ entre 1979 e 1984 para 17,9\% entre 1995 e $2000^{6}$.

Os dados referentes à epidemiologia da sepse no Brasil ainda são escassos, no entanto dois estudos recentes procuraram abordar esta questão. O Bases Study ${ }^{3}$ avaliou 1383 pacientes internados em cinco unidades de terapia intensiva (três em São Paulo e duas em Santa Catarina) durante cinco meses. Do total de 1383 pacientes incluídos, 415 pacientes $(30,5 \%)$ desenvolveram sepse, $241(17,4 \%)$ sepse grave e 203 (14,7\%) choque séptico. A taxa de letalidade encontrada foi de $33,9 \%, 46,9 \%$ e $52,2 \%$, para sepse, sepse grave e choque séptico, respectivamente. Um segundo estudo, Sepse Brasil ${ }^{5}$, incluiu 3128 pacientes em 75 UTI de 65 hospitais, dos quais 521 (16,7\%) foram identificados como sepse, sepse grave ou choque séptico. 
Foi observada letalidade na UTI de $16,7 \%, 34,4 \%$ e $65,3 \%$, respectivamente. O tempo médio de internação do paciente com sepse foi de 15 dias. Vale ressaltar que estes estudos, embora metodologicamente corretos, talvez não reflitam integralmente a realidade brasileira, por incluir majoritariamente pacientes internados em hospitais terciários, situados em sua maioria, nas regiões sudeste e sul do país.

Nesse contexto de morbimortalidade elevada e custos crescentes é fundamental que as sociedades médicas encampem iniciativas que apontem na direção da geração e difusão de conhecimento a respeito da sepse, além de buscar as evidências atuais e promover estudos experimentais, epidemiológicos e ensaios clínicos, assim como, trabalhar pela aplicação da boa prática clínica. A Campanha de Sobrevivência da Sepse (CSS) idealizada em 2002 num esforço conjunto de diversas sociedades internacionais de medicina intensiva e infectologia apontam nessa direção. Iniciada pela chamada declaração de Barcelona (2002), que propôs um esforço internacional para a redução da letalidade da sepse em $25 \%$ em 5 anos. Para tal, foram produzidos documentos com recomendações amplas que vem sendo difundidos com grande empenho. Contudo, passado aproximadamente três anos da publicação das principais recomendações da CSS ${ }^{7}$, nos cabe fazer uma reflexão crítica, acerca das recomendações contidas nesses documentos, com ênfase na reprodutibilidade e potenciais benefícios da aplicação deste conjunto de recomendações em nosso meio.

\section{AS EVIDÊNCIAS, AS RECOMENDAÇÕES E OS PRO- TOCOLOS}

Em função dos dados apresentados, não há dúvidas de que a sepse é hoje um problema de saúde pública, devendo, portanto, ser abordado com medidas ligadas a saúde coletiva, bem como, com medidas voltadas para saúde do paciente. Dentre as medidas coletivas, devem estar incluídas políticas que garantam o acesso dos pacientes ao sistema de saúde, disponibilização de leitos de terapia intensiva de qualidade, conduzidos por especialistas, treinamento dos diferentes níveis do sistema para o diagnóstico e tratamento das principais doenças infecciosas, prevenção de infecções nosocomiais, utilização racional de antibióticos, entre outras. Ao nosso ver a abordagem da CSS se restringe à saúde do paciente, o que evidentemente tem abrangência limitada. Dentre os muitos méritos inerentes a CSS destaca-se a tentativa de basear as recomendações em evidências científicas sólidas, que fundamentem a prática diária da assistência do paciente com sepse. Entretanto, esta proposta parece não se cumprir integralmente e tem se tornado motivo de diversas críticas, que vêm sendo publicadas em periódicos médicos nestes últimos anos $^{8-10}$. As recomendações da CSS adotam um modelo de hierarquização denominada método de Delphi modificado ${ }^{11}$. Neste sistema, dependendo da "qualidade" da evidência, a recomendação é classificada em um dos cinco níveis, de A a E, sendo A (o maior grau) apoiado por grandes estudos "aleatórios". Ao nosso ver, este modelo quando aplicado a situação de abordagens complexas e multidimensionais como a sepse, induz a distorções quanto sua a aplicação e a boa prática clínica. Por exemplo, fornecer um nível de evidência E para a antibioticoterapia e drenagem do foco infeccioso, por não haver estudos aleatórios, é no mínimo um contrasenso. Hoje não é aceitável a realização de um estudo clinico sobre tratamento de sepse com ou sem antibiótico, pois é evidente que a antibioticoterapia é uma "evidência" consolidada. Tal qual não é possível a realização de um ensaio aleatório controlado sobre o impacto na prevenção de trauma ou morte nos saltos com e sem pára-quedas ${ }^{12}$, não é razoável ou plausível discutir sepse sem destacar a proeminência do uso de medidas terapêuticas fundamentais. Entretanto, mesmo compreendendo a utilidade dos estudos controlados e aleatórios para a boa prática clínica, devemos considerar o uso da "melhor evidência, sendo a melhor disponível" não se podendo sofismar impunemente nesse campo. De fato, muitas das recomendações presentes na CSS sequer derivam de estudos realizados em pacientes com sepse tais como a profilaxia de TVP, profilaxia de úlcera de estresse ou controle glicêmico estrito. Se por um lado acreditamos no benefício dessas recomendações para uma população geral de pacientes críticos, nos faltam evidências sólidas da literatura (estudos realizados especificamente com pacientes sépticos) para que estas recomendações possam ter o real peso de evidência imputado a elas pelo documento da CSS. Mais difícil ainda é aceitar que "pacotes" (bundles) de medidas devam ser universalmente implementados em busca de amplificar os benefícios das medidas individuais. Esse aspecto em particular deve ser destacado, uma vez que a soma dos componentes dos bundles pode não ser tão eficaz quanto imaginado, especialmente se considerarmos que estas intervenções não foram testadas em ensaios clínicos adequados.

Em resumo, estamos felizes por haver uma crescente atenção no reconhecimento da sepse como questão 
maior de saúde publica. No entanto, maior ainda será o impacto das políticas voltadas para a redução da mortalidade na sepse se pudermos realizar estudos que nos permitam conhecer melhor a nossa realidade, implementar medidas básicas de garantia do acesso à saúde e treinar os profissionais de saúde para que a boa pratica clínica sejam implementadas. Este papel deve ser assumido pelos profissionais e instituições individualmente e pelas sociedades médicas, fazendo uma interface mais efetiva com a população, as agencias reguladoras e com o Ministério da Saúde. Diminuir as medidas iatrogênicas e zelar pela segurança dos pacientes deve fazer parte dos nossos objetivos primordiais. Nesse sentido a avaliação cautelosa das evidências, a demanda por novos ensaios clínicos e confirmatórios da eficácia e segurança do uso de drogas novas e especialmente daquelas que fazem parte do nosso arsenal terapêutico atual tais como corticosteróides e drotrecogina alfa é essencial. Do contrário, ao buscarmos incessantemente os benefícios de cada paciente podemos incorrer na generalização de um conjunto de verdades transitórias e não validadas, e muitas não já o foram?. E voltando a Brás Cubas: "Não me podes negar um fato: o prazer do beneficiador é sempre maior que o do beneficiado. Que é o benefício?"

\section{REFERÊNCIAS}

01. Russell JA - Management of sepsis. N Engl J Med, 2006;355:16991713.

02. Angus DC, Linde-Zwirble WT, Lidicker $\mathrm{J}$ et al - Epidemiology of severe sepsis in the United States: analysis of incidence, outcome, and associated costs of care. Crit Care Med, 2001;29:1303-1310.

03. Silva E, Pedro Mde A, Sogayar AC et al - Brazilian Sepsis Epidemiological Study (BASES study). Crit Care, 2004;8:R251-R260.

04. Vincent JL, Sakr Y, Sprung CL et al - Sepsis in European intensive care units: results of the SOAP study. Crit Care Med, 2006;34:344-353.

05. Sales Jr JA, David CM, Hatum R et al - Sepse Brasil: estudo epidemiológico da sepse em unidades de terapia intensiva brasileiras. RBTI, 2006;18:9-17.

06. Martin GS, Mannino DM, Eaton S et al - The epidemiology of sepsis in the United States from 1979 through 2000. N Engl J Med, 2003;348:15461554.

07. Dellinger RP, Carlet JM, Masur $\mathrm{H}$ et al - Surviving Sepsis Campaign Management guidelines for management of severe sepsis and septic shock. Crit Care Med, 2004;32:858-873.

08. Landucci D - The surviving sepsis guidelines: "lost in translation". Crit Care Med, 2004;32:1598-1600.

09. Singer M - The Surviving Sepsis guidelines: evidence-based ... or evidence-biased? Crit Care Resusc, 2006;8:244-245.

10. Eichacker PQ, Natanson C, Danner RL - Surviving sepsis--practice guidelines, marketing campaigns, and Eli Lilly. N Engl J Med, 2006;355:16401642.

11. Sackett DL - Rules of evidence and clinical recommendations on the use of antithrombotic agents. Chest, 1989;95:(Suppl2)2S-4S.

12. Smith GC, Pell JP - Parachute use to prevent death and major trauma related to gravitational challenge: systematic review of randomised controlled trials. Int J Prosthodont, 2003;19:126-128. 